University of Wollongong

Research Online

Australian Institute for Innovative Materials -

Papers

Australian Institute for Innovative Materials

$1-1-2014$

Nonlinear optical conductivity of two-dimensional semiconductors with

Rashba spin-orbit coupling in terahertz regime

Yee Sin Ang

University of Wollongong, ysa190@uowmail.edu.au

J C. Cao

Chinese Academy of Sciences

Chao Zhang

University of Wollongong, czhang@uow.edu.au

Follow this and additional works at: https://ro.uow.edu.au/aiimpapers

Part of the Engineering Commons, and the Physical Sciences and Mathematics Commons

Research Online is the open access institutional repository for the University of Wollongong. For further information contact the UOW Library: research-pubs@uow.edu.au 


\title{
Nonlinear optical conductivity of two-dimensional semiconductors with Rashba spin-orbit coupling in terahertz regime
}

\begin{abstract}
We reveal that two-dimensional semiconductors with Rashba spin-orbit interaction (R2DG) exhibit exceptionally strong nonlinear optical response (NOR) in the terahertz frequency regime. The spin-split of the parabolic energy band in R2DG allows strong multiple-photon process to occur via inter-subband mechanism. We show sharp multiple photon edges in the nonlinear conductivity. The edges correspond to the cut-off effect produced by the multiple-photon process. For Rashba coupling parameter of $\lambda \mathrm{R} \approx$ 10-10 eV m, electric field strength in the order of only $102 \mathrm{~V} / \mathrm{cm}$ is required for the NOR to dominate over the linear response. Furthermore, the roles of the parabolic 'free electron' term $\mathrm{H} 0$ and the linear Rashba term $\mathrm{H}$ R on NOR of R2DG are also investigated. Although the NOR is made possible due to the presence of a finite $\mathrm{HR}, \mathrm{H} O$ does play an important role on the NOR especially in high temperature regime. $\mathrm{H} \mathrm{O}$ has rendered R2DG a strong optical nonlinearity at elevated temperature which is not found in a purely linear system such as graphene. The results suggest the possibilities of Rashba spintronic system in the application of nonlinear terahertz devices.
\end{abstract}

\section{Keywords}

nonlinear, optical, terahertz, conductivity, regime, coupling, spin, two, dimensional, semiconductors, rashba, orbit

\section{Disciplines}

Engineering | Physical Sciences and Mathematics

\section{Publication Details}

Ang, Y., Cao, J. C. \& Zhang, C. (2014). Nonlinear optical conductivity of two-dimensional semiconductors with Rashba spin-orbit coupling in terahertz regime. European Physical Journal B: Condensed Matter and Complex Systems, 87 (28), 1-7. 


\title{
Nonlinear optical conductivity of two-dimensional semiconductors with Rashba spin-orbit coupling in terahertz regime
}

\author{
Yee Sin Ang \\ School of Physics, University of Wollongong, Wollongong, NSW 2522, Australia
}

J. C. Cao

Key Laboratory of Terahertz Solid State Technology,

Shanghai Institute of Microsystem and Information Technology, Chinese Academy of Sciences, Shanghai, China

\author{
Chao Zhang* \\ School of Physics and Institute for Superconducting and Electronic Materials, \\ University of Wollongong, Wollongong, NSW 2522, Australia
}

\begin{abstract}
We show that two-dimensional semiconductors with Rashba spin-orbit interaction (R2DG) exhibits exceptionally strong nonlinear optical response (NOR) in the terahertz frequency regime. The spin-splitting of the parabolic energy band in R2DG allows strong multiple-photon process to occur via inter-subband mechanism. We observe multiple sharp edges in the nonlinear conductivity frequency spectrum. The edges correspond to a cut-off effect produced by the multiple-photon process. For Rashba coupling parameter of $\lambda_{R} \approx 10^{-10} \mathrm{eVm}$, electric field strength in the order of only $10^{2} \mathrm{~V} / \mathrm{cm}$ is required for the NOR to dominate over the linear response. Furthermore, the roles of the parabolic 'free electron' term $H_{0}$ and the linear Rashba term $H_{R}$ on NOR of R2DG are also investigated. Although the NOR is solely made possible due to the presence of a finite $H_{R}, H_{0}$ does play an important role on the NOR especially in high temperature regime. $H_{0}$ has rendered R2DG a strong optical nonlinearity at elevated temperature which is not found in a purely linear system such as graphene. The results suggest the possibilities of Rashba spintronic system in the application of nonlinear terahertz devices.
\end{abstract}

PACS numbers: 71.70.Ej, 73.21.Fg, 72.30.+q

\section{INTRODUCTION}

Following the advancement of spintronics ${ }^{1-5}$, twodimensional electronic system with lifted spin-degeneracy has gained much attentions. It is possible to create finite spin-orbit interaction and to lift the spin-degeneracy of a two-dimensional electronic system spontaneously in the absence of an external magnetic field. In bulk material with inversion asymmetry of the crystal structure, SOI results in the lift of the spin-degeneracy intrinsically via Dresselhaus SOI mechanism ${ }^{6}$. For two-dimensional electrons, such as an electron gas confined in a quantum well structure (QWS) and surface states of metals, the electrons can undergo spontaneous lifting of the spindegeneracy if the confining potential is asymmetric. Such effect is equivalent to the relativistic case of electron moving through a surface with inhomogeneous electric field. In the rest frame of electrons, the electric field is relativistically equivalent to a magnetic field. This generates finite SOI which energetically separating the electron gas into two populations of different chirality. SOI of this form is known is the Rashba spin-orbit interaction $(\mathrm{RSOI})^{7}$. The RSOI manifests itself as a left-and rightshifting of the 'free' electron parabolic bands in phasespace, characterized by Rashba coupling parameter $\lambda_{R}$. The Rashba effect in QWS is especially interesting since the RSOI strength can be tunable via external gating.

Recently, strong nonlinear optical response (NOR) of graphene and several sister-structures have been reported $^{8-13}$. The strong optical nonlinearity of graphene originates from the massless Dirac fermions residing at the vicinity of $K$-point in the first Brillouin zone ${ }^{14-16}$. The R2DG shares a similarity with $K$-point massless Dirac Fermions in graphene. The motion of massless Dirac Fermion in graphene is coupled to its pseudospin while in R2DG electron motion is coupled to real spin. In graphene, the psedospin-momentum coupling manifest itself as a Dirac Hamiltonian linear in $k$-space $H_{K}=v_{F} \vec{\sigma} \cdot \vec{p}$ while in R2DG the real spin-momentum coupling manifest itself as a Rashba Hamiltonian $H_{R}=v_{R}(\vec{\sigma} \times \vec{p})_{z}$ which is also linear in momentum space. The Fermi velocity $v_{F}$ is about $10^{6} \mathrm{~m} / \mathrm{s}$ in graphene and $v_{R}=\lambda_{R} / \hbar$ is 'Rashba velocity' in R2DG. Due to the linear form of $H_{K}$ and $H_{R}$, strong terahertz four-wave mixing has been predicted in both graphene and R2DG via intrasubband mechanism ${ }^{12,17}$. Such nonlinear photon-mixing effect occurs because the linear term is rich in higher order harmonics when the electrons are perturbed by an external electric field.

The spin-splitting of the parabolic band in R2DG creates additional pathway for optical transition via intersubband mechanism. While the inter-subband linear optical response of R2DG has been studied in detail ${ }^{18,19}$, the inter-subbband NOR of R2DG remains unknown. In graphene, the inter-subband (optical transition between lower and upper branches of the Dirac cone) NOR is huge due to the largeness of $v_{F}\left(\mathrm{NOR} \propto v_{F}^{2}\right.$ as reported $\left.\mathrm{in}^{8}\right)$. Therefore, in order to achieve experimen- 
tally observable inter-subband NOR in R2DG, $v_{R}$ (and hence $\lambda_{R}$ ) has to be sufficiently large. Although a rather large $\lambda_{R} \approx 4 \times 10^{-11} \mathrm{eVm}$ has been achieved in $\mathrm{In}_{0.53} \mathrm{Ga}_{0.47} / \mathrm{In}_{0.52} \mathrm{Al}_{0.48} \mathrm{As} \mathrm{QWS}^{20,21}$, such RSOI corresponds to $v_{R}$ of only $6 \times 10^{4} \mathrm{~m} / \mathrm{s}$, which is significantly slower than $v_{F}$ in graphene. The NOR of this R2DG system is expected to be approximately $10^{4}$ times smaller than graphene.

Several recent experimental work on BiTeI layered semiconductor and $\mathrm{Bi} / \mathrm{Ag}(111)$ surface has achieved giant RSOI in the order of $\lambda_{R} \approx 10^{-10} \mathrm{eVm}^{22,23}$. The one-order of magnitude increment of $\lambda_{R}$ immediately suggests the possibility of generating strong NOR comparable to that found in graphene. Motivated by these experimental results, we theoretically investigated the intersubband NOR of R2DG in terahertz frequency regime. By performing a Floquet analysis on the electron-photon coupled R2DG system ${ }^{24-26}$, we derived the light-dressed states of R2DG in the presence of an external electric field and determined the nonlinear optical conductivities of R2DG up to third-order in the external field. For a typical $\lambda_{R}$ in the order of $10^{-11} \mathrm{eVm}$, the required electric fields for NOR to dominate over the linear response at $1 \mathrm{THz}$ is only in the order of $10^{2} \mathrm{~V} / \mathrm{cm}$. We also investigate the roles of electron effective mass $m^{*}$ and $\lambda_{R}$ on NOR. The R2DG is actually a mixture of non-relativistic 'free' electron and relativistic massless Dirac fermions with the non-relativistic parabolic component $H_{0}$ characterized by $m^{*}$ and the linear $H_{R}$ relativistic component characterized by $\lambda_{R}$. Interestingly, the R2DG's NOR, which is made possible due to the presence of finite $H_{R}$, depends not only on $\lambda_{R}$, but also on $m^{*}$. Using recent giant RSOI experimental values of $\lambda_{R}{ }^{22,23}$, we compare optical nonlinearity in R2DG with that of graphene, one of the strongest nonlinear materials known to-date ${ }^{8,9}$. At high temperature regime, optical nonlinearity of such Rashba systems can be three times stronger than that of graphene. This clearly suggests a potential of Rashba spintronic system in room temperature nonlinear terahertz application.

\section{FORMALISM}

In this section, we derive the photon-dressed electronic wavefunctions of R2DG under an external field and describe the general recipe to calculate optical current density. We consider a two-dimensionally confined electron gas in $x$ - $y$ plane with RSOI (such as asymmetrically confined electron gas in InAs quantum well ${ }^{20,21}$ or surface states of metals, semiconductor and surface alloys $^{22,27,28}$ ). We consider the intrinsic case where electrons occupies only the $s=-1$ band with Fermi level sit- uated at the band-crossing point at $T=0 \mathrm{~K}$ (i.e. Fermi level $\left.\varepsilon_{F}=0\right)$. The Hamiltonian is a combination of a parabolic free electron term $H_{0}$ and a linear Rashba term $H_{R}$,

$$
\begin{aligned}
\mathcal{H}(p) & =H_{0}(p)+H_{R}(p) \\
& =\alpha\left[\begin{array}{cc}
p_{x}^{2}+p_{y}^{2} & 0 \\
0 & p_{x}^{2}+p_{y}^{2}
\end{array}\right]+v_{R}\left[\begin{array}{cc}
0 & p_{y}+i p_{x} \\
p_{y}-i p_{x} & 0
\end{array}\right]
\end{aligned}
$$

where $\alpha=1 / 2 m^{*}$ and $v_{R}=\lambda_{R} / \hbar . m^{*}$ and $\lambda_{R}$ are the free electron effective mass and Rashba coupling parameter respectively. $\alpha$ and $v_{R}$ are related to the Rashba splitting energy via $E_{R}=v_{R}^{2} / 4 \alpha . p_{x}$ and $p_{y}$ denotes the $x$-and $y$-directional momentum component respectively. The Hamiltonian of R2DG in phase space representation is similar to that of the bilayer graphene (BLG) with low energy trigonal warping, except that in R2DG the parabolic term is diagonal while in BLG the parabolic term is off-diagonal ${ }^{29,30}$. The eigenvalue is given as

$$
\varepsilon_{s}=\alpha p^{2}+s v_{R} p
$$

where $s= \pm 1$ denoting the subband index as a result of Rashba spin-splitting. The band structure is shown in Fig. 1(a). The eigenfunction is

$$
\xi_{0}(p)=\frac{1}{\sqrt{2}}\left[\begin{array}{c}
s p_{+} / p \\
1
\end{array}\right]
$$

where $p_{ \pm}=p_{y} \pm i p_{x}$ and $p=\left(p_{x}^{2}+p_{y}^{2}\right)^{1 / 2}$. We consider an external electric field in the form of $\mathbf{E}=\hat{x} E e^{i \omega t}$ along the $x$-direction. The vector potential is given as $\mathbf{A}=\mathbf{E} / i \omega$. We can minimally couple the electron to the photon via $p_{x} \rightarrow p_{x}+e A$. The electron-photon interaction Hamiltonian can be obtained by the substitution $\mathcal{H}^{\prime}=\mathcal{H}\left(p_{x} \rightarrow p_{x}+e A\right)$. Since $\mathcal{H}^{\prime}$ is time-periodic, the light-dressed electronic wavefunction can be expressed as a linear combination of Floquet states

$$
\Psi(p)=\sum_{n=0}^{\infty} e^{i\left(n \omega-\varepsilon_{+} / \hbar\right) t} \psi_{n}(p),
$$

where $\psi_{n}(p)=\left(a_{n}(p), b_{n}(p)\right)^{T}$ is the Floquet states. It is composed of the spinor components $a_{n}(p)$ and $b_{n}(p)$, each representing $n$-photon coupling of electron. By solving the Schroedinger equation $i \hbar \partial \Psi(p) / \partial t=\mathcal{H}^{\prime} \Psi(p)$, we obtain a set of recursive equations connecting $n$ th-order spinor components with $(n-1)$ th order spinor components 


$$
\begin{aligned}
a_{n} & =\frac{1}{n \hbar \omega\left(n \hbar \omega-2 v_{R} p\right)}\left\{-\mathcal{F}\left[2 i \alpha\left(v_{R} p-n \hbar \omega\right) p_{x}+v_{R}^{2} p_{+}\right] a_{n-1}+v_{R} \mathcal{F}\left[\left(v_{R} p-n \hbar \omega\right)-2 i \alpha p_{x} p_{+}\right] b_{n-1}\right. \\
& \left.-\alpha \mathcal{F}^{2}\left(v_{R} p-n \hbar \omega\right) a_{n-2}-\alpha v_{R} \mathcal{F}^{2} p_{+} b_{n-2}\right\}, \\
b_{n} & =\frac{1}{n \hbar \omega\left(n \hbar \omega-2 v_{R} p\right)}\left\{-\mathcal{F}\left[2 i \alpha\left(v_{R} p-n \hbar \omega\right) p_{x}-v_{R}^{2} p_{-}\right] b_{n-1}-v_{R} \mathcal{F}\left[\left(v_{R} p-n \hbar \omega\right)+2 i \alpha p_{x} p_{-}\right] a_{n-1}\right. \\
& \left.-\alpha \mathcal{F}^{2}\left(v_{R} p-n \hbar \omega\right) b_{n-2}-\alpha v_{R} \mathcal{F}^{2} p_{-} a_{n-2}\right\},
\end{aligned}
$$

where $\mathcal{F}=e E / \omega$. By recursively applying Eq. (5) and Eq. (6), we can construct spinor components to any order in electron-photon coupling. The recursive nature of the spinor components originates from the off-diagonal $H_{R}$. In fact, the off-diagonality of the Hamiltonian is a fun- damental consequence due to the subband spin-splitting in a two-dimensional system. For $n=0$, there is no photon. The zero-order spinor components are found to be $a_{0}=p_{+} / \sqrt{2} p$ and $b_{0}=1 / \sqrt{2}$, respectively, which is in agreement with the single electron eigenfunction Eq. (3). For $n=1$, the spinor components are

$$
\begin{aligned}
& a_{1}=\frac{\mathcal{F}}{\sqrt{2} \hbar \omega\left(\hbar \omega-2 v_{R} p\right)}\left\{-2 i \alpha\left(v_{R} p-\hbar \omega\right) \frac{p_{+}}{p}+v_{R}\left[\left(v_{R} p-\hbar \omega\right)-2 i \alpha p_{x} p_{+}\right]\right\}, \\
& b_{1}=\frac{\mathcal{F}}{\sqrt{2} \hbar \omega\left(\hbar \omega-2 v_{R} p\right)}\left\{-2 i \alpha\left(v_{R} p-\hbar \omega\right)-v_{R}\left[\left(v_{R} p-\hbar \omega\right) \frac{p_{+}}{p}++2 i \alpha p_{x} p\right]\right\},
\end{aligned}
$$

where $\omega=\omega-i 0$. The frequency-dependent current density can be calculated from

$$
J=-e \Re \int p \mathrm{~d} p \mathrm{~d} \phi \hat{j} \mathcal{N}\left(v_{R} p\right),
$$

where $\Re$ denotes real part, $\hat{j}=\Psi^{\dagger}(p) \hat{v}_{x} \Psi(p)$ is the current operator and $\hat{v}_{x}=\partial \mathcal{H}\left(p_{x}+e A\right) / \partial p_{x}$ is the velocity operator

$$
\hat{v}_{x}=\left[\begin{array}{cc}
2 \alpha\left(p_{x}+e A\right) & i v_{R} \\
-i v_{R} & 2 \alpha\left(p_{x}+e A\right)
\end{array}\right] .
$$

The temperature dependence of $J$ is contained in $\mathcal{N}\left(v_{R} p\right)=f\left(\varepsilon_{-}\right)-f\left(\varepsilon_{+}\right)$where $f\left(\varepsilon_{ \pm}\right)$is the Fermi-Dirac distribution function.

\section{LINEAR OPTICAL RESPONSE}

For linear optical response, the current operator is simply the overlap between $n=0$ spinor components and $n=1$ spinor components over the velocity operator, i.e.

$$
\hat{j}_{1}=\left(\psi_{0}^{\dagger} \hat{v}_{x} \psi_{1}+\psi_{1}^{\dagger} \hat{v}_{x} \phi_{0}\right) e^{i \omega t},
$$

where $\psi_{n}=\left(a_{n}, b_{n}\right)^{T}$. We can apply the Dirac's identity to eliminate the momentum integration in Eq. (9). Integrating the remaining angular part, we obtain linear optical conductivity

$$
\sigma_{1}(\omega)=\frac{e^{2}}{8 \hbar} \mathcal{N}(\hbar \omega / 2)
$$

which is consistent with the results calculated using Kubo formalism ${ }^{18}$. The linear conductivity is shown in Fig. 1. At $T=0 \mathrm{~K}$, the linear conductivity is a square function of incident photon frequency. The linear conductivity becomes zero when a maximum frequency $\hbar f_{\max }=4 v_{R}^{2} / \alpha=8 E_{R}$ is exceeded. This happens because the energy spacing between $s=+1$ and $s=-1$ subbands is insufficient to accommodate high frequency optical transition of the electron [see Fig. 1(a)]. For $E_{R}=2.1 \mathrm{meV}, f_{\max } \approx 4 \mathrm{THz}$. At $T=77 \mathrm{~K}$, states in $s=-1$ subband with energy $E>0$ are thermally populated and also contributes to the linear conductivity. These results in the smearing of the linear conductivity up to about $f=10 \mathrm{THz}$. 
(a)
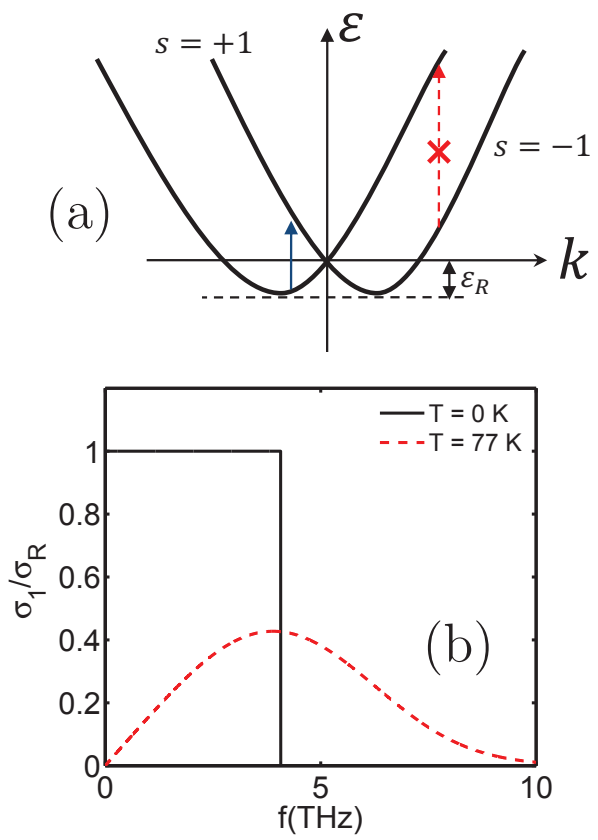

FIG. 1. (Color online) (a) Band structure of R2DG. The Fermi energy is at $\varepsilon_{F}=0$. The red (dashed) arrow denote forbidden interband transition. The blue (solid) arrow denote permissible interband transition; (b) Linear conductivity. Rashba energy of $E_{R}=2.1 \mathrm{meV}$ is used. The conductivity is normalized by $\sigma_{R}=e^{2} / 8 \hbar$.

\section{THIRD-ORDER NONLINEAR OPTICAL RESPONSE}

The third-order nonlinear current operator consists of two components: (i) a single-frequency term; and (ii) triple-frequency term. The single-frequency term originates from the overlapping between $\psi_{2}$ and $\psi_{1}$ which carries an oscillating part of $e^{i \omega t}$; while the triple-frequency term originates from the overlapping between $\psi_{3}$ and $\psi_{0}$ which carries an oscillating part of $e^{3 i \omega t}$. Explicitly, they are given as

$$
\begin{gathered}
\hat{j}_{3}(\omega)=\left(\psi_{2}^{\dagger} \hat{v}_{x} \psi_{1}+\psi_{1}^{\dagger} \hat{v}_{x} \psi_{2}\right) e^{i \omega t} \\
\hat{j}_{3}(3 \omega)=\left(\psi_{3}^{\dagger} \hat{v}_{x} \psi_{0}+\psi_{0}^{\dagger} \hat{v}_{x} \psi_{3}\right) e^{i 3 \omega t}
\end{gathered}
$$

The third-order nonlinear optical conductivity can then be obtained by evaluating Eq. (9). The single-frequency and triple-frequency conductivities are given, respectively, as

$$
\sigma_{3}(\omega)=\sigma_{R} \frac{e^{2} E^{2}}{\hbar \omega^{3}}\left[\frac{v_{R}^{2}}{\hbar \omega}(2+x) \mathcal{N}(\hbar \omega)+\frac{\alpha}{8} \mathcal{N}\left(\frac{\hbar \omega}{2}\right)\right]
$$

$$
\sigma_{3}(3 \omega)=\sigma_{R} \frac{e^{2} E^{2}}{\hbar \omega^{3}}\left[\frac{v_{R}^{2}}{\hbar \omega}\left(g_{1}+g_{2}+g_{3}\right)+\alpha\left(g_{4}+g_{5}\right)\right]
$$

where $\sigma_{R}=e^{2} / 8 \hbar$ and

$$
\begin{aligned}
x & =\frac{\hbar \omega}{4 E_{R}} \\
g_{1} & =\left(\frac{13}{48}+\frac{x}{8}+\frac{x^{2}}{2}\right) \mathcal{N}\left(\frac{\hbar \omega}{2}\right) \\
g_{2} & =\left(-\frac{2}{3}+2 x\right) \mathcal{N}(\hbar \omega) \\
g_{3} & =\frac{15}{16} \mathcal{N}\left(\frac{3 \hbar \omega}{2}\right) \\
g_{4} & =-\frac{1}{3} \mathcal{N}\left(\frac{\hbar \omega}{2}\right) \\
g_{5} & =\left(\frac{1}{6}+x\right) \mathcal{N}\left(\frac{3 \hbar \omega}{2}\right) .
\end{aligned}
$$

\section{A. Frequency and temperature dependence}

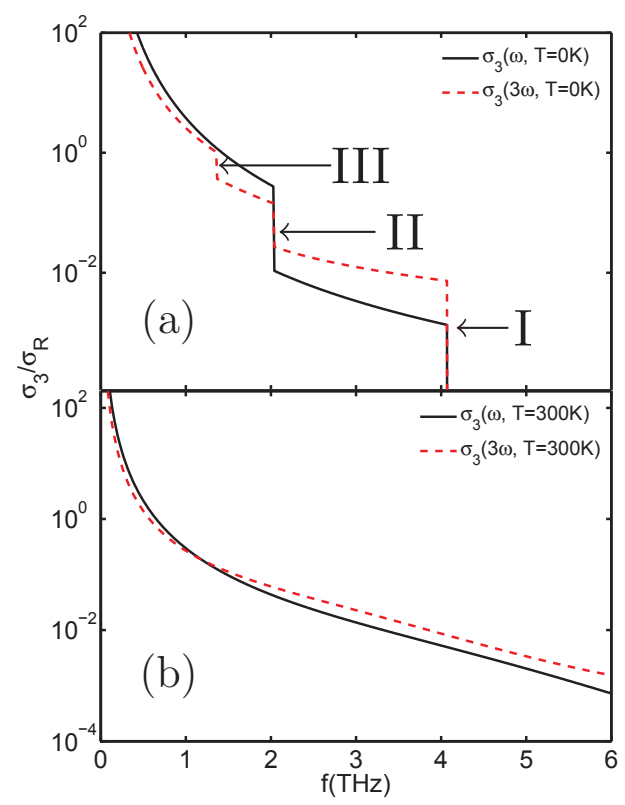

FIG. 2. (Color online) Third order nonlinear optical conductivities $\sigma(\omega)$ (solid curve) and $\sigma(3 \omega)$ (dashed curve) at $E=1000 \mathrm{~V} / \mathrm{cm}$. (a) $T=0 \mathrm{~K}$, the sharp edges marks the cut-off of 1-photon edge (I), 2-photon edge (II) and 3-photon edge (III); (b) $T=300 K$.

The frequency dependence of the third-order nonlinear conductivities is shown in Fig. 2. We have chosen a moderate Rashba splitting energy of $E_{R}=2.1 \mathrm{meV}$ and 'Rashba velocity' of $v_{R}=5.026 \times 10^{4} \mathrm{~m} / \mathrm{s}^{27}$. At $T=0 \mathrm{~K}$

and 
three step-like edges are clearly observable for both $\sigma(\omega)$ and $\sigma(3 \omega)$. These steps are related to the existence of three distinct Dirac delta functions $\delta\left(n \hbar \omega-2 v_{R} p\right)$ with $n=1,2,3$ when evaluating the optical current integral in Eq. (9). The separation between $s=+1$ and $s=-1$ subbands at a fixed $\hbar \omega$ is $\Delta=\varepsilon_{+}-\varepsilon_{-}=2 v_{R} p$. The delta functions therefore physically represents the cut-off of an optical process when electron absorb $n$-photon to transit from $s=-1$ to $s=+1$ subband is no longer possible. At low temperature, electrons are mostly residing in $s=-1$ subband. Since the maximum permissible frequency-width for interband transition is $h f_{\max }=8 \varepsilon_{R}$, $\delta\left(n \hbar \omega-2 v_{R} p\right)$ truncates any n-photon process at $\omega_{\max } / n$, giving rise to a ' $n$-photon edge' as shown in Fig. 2(a). Albeit the similarity of graphene's $H_{K}$ and R2DG's $H_{R}$, such truncation does not occur in graphene because in graphene the $s=-1$ subband does not 'bend-up' as electron wavevector $k$ increases. There is no $f_{\max }$ and hence the permissible frequency for optical absorption can increases as far as the linear Dirac-cone approximation still holds. The $T=300 \mathrm{~K}$ nonlinear conductivities are plotted in Fig. 2(b). At higher temperature, the thermal excitation of electrons to higher lying states washes out the $n$-photon edges. At low frequency, the NOR is initially large but decreases with temperature since low lying conducting states are thermally depopulated. At higher frequency, the NOR however behaves in a contrasting way: it is initially small and increases with temperature. This is because the $s=-1$ electrons are thermally excited to states with higher energy and are capable of performing large-frequency interband transition. The optical response of R2DG is hence sensitively influenced by temperature. At approximately $f>1 \mathrm{THz}$, the $\sigma_{3}(3 \omega)>\sigma_{3}(\omega)$, signifying a stronger frequency tripling effect is taking place.

\section{B. Critical electric field strength}

The nonlinear conductivities $\sigma_{3}(\omega)$ and $\sigma_{3}(3 \omega)$ alone are insufficient to determine whether a material is strongly nonlinear since the linear response can be much stronger and masks out the NOR. To quantify the degree of optical nonlinearity, we define critical electric field strength $E_{c}$ as the required field strength to achieve $\sigma_{3}=\sigma_{1}$. Small $E_{c}$ signifies strong optical nonlinearity. The temperature dependence of the single-frequency NOR $E_{c}(\omega)$ and triple-frequency NOR $E_{c}(3 \omega)$ at $\mathrm{THz}$ frequency regime is shown in Fig. 3. At low frequency $(f=1 \mathrm{THz}), E_{c}$ decreases with increasing temperature since $\sigma_{1}$ decreases very rapidly as a result of its stronger temperature dependence in comparison with the nonlinear terms [Fig. 3(a)]. At $f=2$ [Fig. 3(b)] THz, a sharp $E_{c}$-peak at low temperature regime can be seen. This happens because $f=2 \mathrm{THz}$ is situated at the vicinity of the 2-photon edge. As temperature increases from zero, smearing of the 2-photon edge decreases the NOR, resulting in an initial rapid rise of $E_{c}$. As the temperature

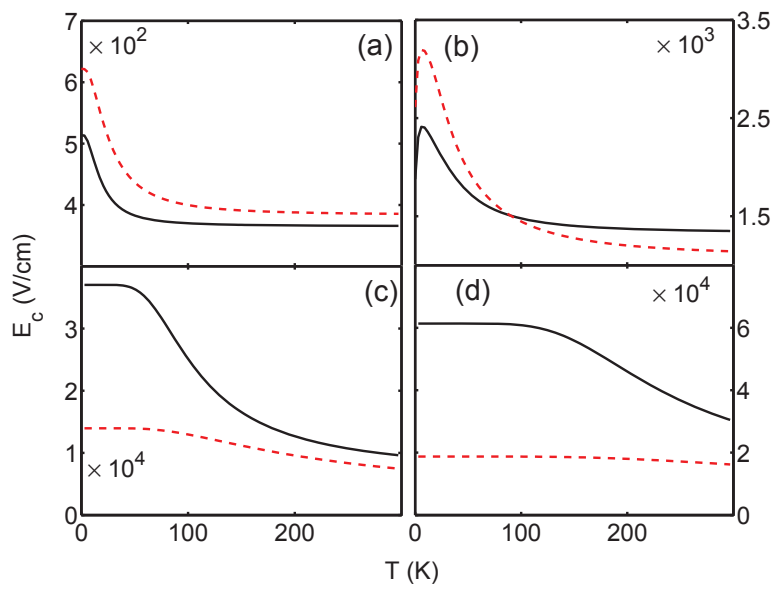

FIG. 3. (Color online) Temperature dependence of critical electric field strength $E_{c}(\omega)$ (solid curve) and $E_{c}(3 \omega)$ (dashed curve) at various frequency: (a) $1 \mathrm{THz}$, (b) $2 \mathrm{THz}$, (c) 5 $\mathrm{THz}$, and (d) $7 \mathrm{THz}$. The low temperature nonlinear conductivities are mainly contributed by 1-photon edge. At higher temperature, the smearing of 2-photon and 3-photon edges enhances the NOR, resulting in gradually decreasing critical field strength.

is further increased, smearing of the 3-photon edge replenishes the NOR and this effectively reduces $E_{c}$, giving rise to the observed $E_{c}$-peak. At high frequency regime $[f=5 \mathrm{THz}$ in Fig. 3(c) and $f=7 \mathrm{THz}$ in Fig. 3(d)], the $E_{c}$ behaves very differently. A low-temperature plateau regime occurs before the gradual decrease of $E_{c}$. This can be explained by the fact that when $f>f_{\max }$ (which is about $4 \mathrm{THz}$ in our case), the low temperature nonlinear conductivities are mainly contributed by the smearing of the 1-photon edge, which, coincidently, has the same $\mathcal{N}(\hbar \omega / 2)$ thermal factor as $\sigma_{1}(\omega)$. The temperature dependence of $E_{c}$, which is the ratio of linear and nonlinear term, is therefore a constant. As temperature increases, the smearing of 2-photon and 3-photon edges extend towards higher frequencies. This enhances the NOR, resulting in the gradual decrease of $E_{c}$ at higher temperature. Interestingly, for $f<5 \mathrm{THz}$, both $E_{c}(\omega)$ and $E_{c}(3 \omega)$ show very little variation as $T>150 \mathrm{~K}$. Therefore, in few $\mathrm{THz}$ regime, the optical nonlinearity of R2DG relative to its linear response is very stable as $T$ increases towards room temperature.

\section{EFFECTS OF $v_{R}$ AND $m^{*}$ ON R2DG NONLINEAR OPTICAL RESPONSE}

In this section, the effects of RSOI coupling parameter $\lambda_{R}$ and electron effective mass $m^{*}$ on R2DG's NOR is discussed. $\lambda_{R}$ and $m^{*}$ represents the relative weighting of parabolic 'free' electron term $H_{0}$ and linear $H_{R}$ term in the R2DG Hamiltonian Eq. (1). Varying $\lambda_{R} \mathrm{~m}$ (or equivalently $v_{R}$ ) and $m^{*}$ is essentially equivalent to tuning the R2DG into a non-relativistic, 'free' electron-like system 
(dominated by $H_{0}$ ) or into a relativistic, massless Dirac fermion-like system (dominated by $H_{R}$ ). The $v_{R}$ of the nonlinear conductivities are plotted for $T=0 \mathrm{~K}$ in Fig. 4(a) and for $T=300 \mathrm{~K}$ for Fig. 4(b) (with $f=1 \mathrm{THz}$, $E=1000 \mathrm{~V} / \mathrm{cm}$ and the effective mass is $\left.m^{*}=0.05 m_{e}\right)$. As $v_{R} \rightarrow 0$ (i.e. RSOI is completely absent), the NOR is completely removed, as is evident in Eq. (14) and (15). At $T=0 \mathrm{~K}$, the $\mathrm{n}$-photon edges again occurs. Since $\omega_{\max } \propto v_{R}, v_{R}$ has to be sufficiently large in order to produce subband-splitting of adequate frequency-width to accommodate the electronic transition from $s=-1$ to $s=+1$ subband. The first edge corresponds to the onset of single-photon process. Multiple-photon processes become possible as $v_{R}$ increases, creating the subsequent 2and 3-photon edges. At $T=300 \mathrm{~K}$, the edges are washed out due to thermal excitation. The smeared edges shifts towards smaller $v_{R}$ since thermally excited electrons allows the onset of the photon-edges at smaller $v_{R}$. The $v_{R}$ dependence of $E_{c}$ at $T=0 \mathrm{~K}$ and $T=300 \mathrm{~K}$ are plotted in Fig. 4(c) and Fig. 4(d) respectively. Note that $E_{c}$ is undefined in the yellow-shaded region the figures since both linear and nonlinear responses are strictly zero when $v_{R}$ is too small. $E_{c}$ is, in general, smaller when $v_{R}$ is large when the RSOI is strong.

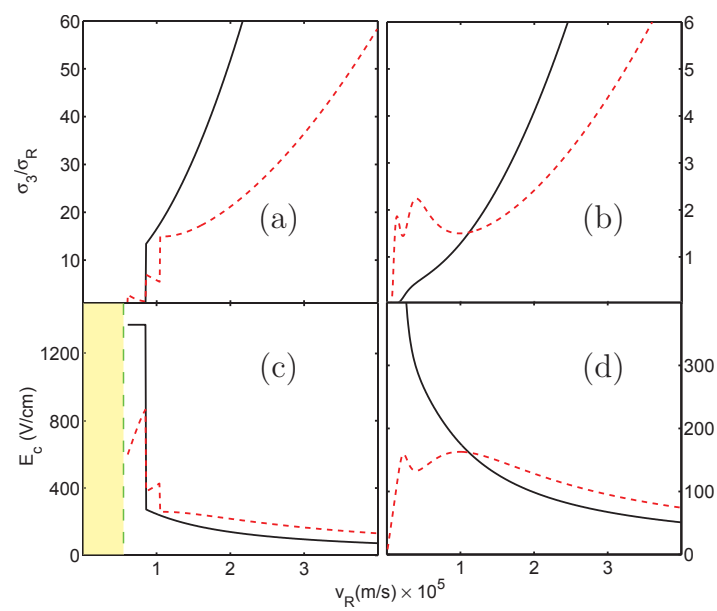

FIG. 4. (Color online) $v_{R}$-dependence of the nonlinear conductivities, $\sigma_{3}(\omega)$ (solid curve) and $\sigma_{3}(3 \omega)$ (dashed curve), at: (a) $T=0 \mathrm{~K}$; (b) $T=300 \mathrm{~K}$ with $E=1000 \mathrm{~V} / \mathrm{cm}$. $v_{R^{-}}$ dependence of the critical electric fields, $E_{c}(\omega)$ (solid curve) and $E_{c}(3 \omega)$ (dashed curve), at: (c) $T=0 \mathrm{~K}$; and (d) $T=300 \mathrm{~K}$. The frequency is set to $1 \mathrm{THz}$ and effective mass $m^{*}=0.05 m_{e}$. The shaded region in the (a) and (c) denotes the regime when the Rashba splitting is too small to accommodate the optical transition of electrons.

Unlike $v_{R}$ which is externally tunable, the effective electron mass $m^{*}$ is an intrinsic properties of the crystal structure and cannot be tuned externally. However, it is still constructive to study the $m^{*}$ dependency of the NOR in order to understand the nonlinear behavior of R2DG. Although an explicit evaluation of Eq. (13) shows that the $\alpha=1 / 2 m^{*}$ terms carried over from the velocity operator $\hat{v}_{x}$ is zero, $m^{*}$ does contained implic-

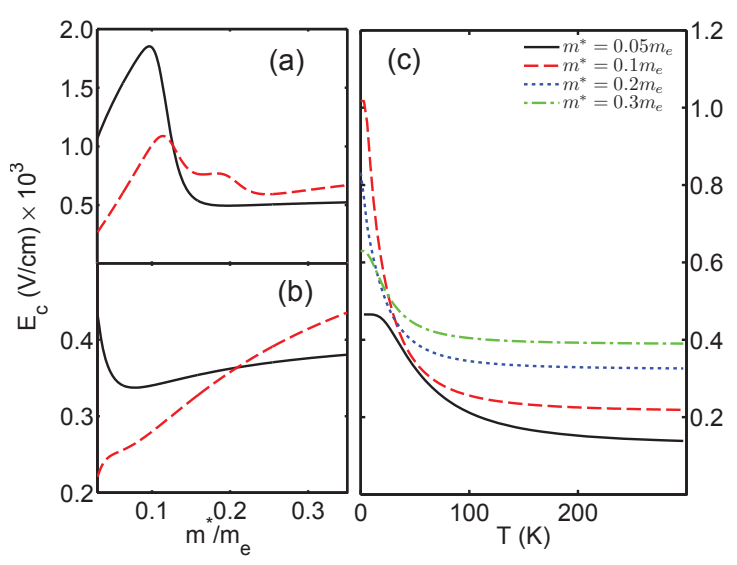

FIG. 5. (Color online) $m^{*}$ dependence of the critical electric field strengths, $E_{c}(\omega)$ (solid curve) and $E_{c}(3 \omega)$ (dashed curve), of the frequency tripling term at $1 \mathrm{THz}$ : (a) $T=4$ $\mathrm{K}$ and (b) $T=15 \mathrm{~K}\left(v_{R}=5 \times 10^{4} \mathrm{~m} / \mathrm{s}\right)$. (c) Temperature dependence of $E_{c}(3 \omega)$ at various values of $m^{*}$. At low temperature, $E_{c}(3 \omega)$ is a complicated function of $m^{*}$ but is, in general, smaller for larger $m^{*}$ (except $m^{*}=0.05 m_{e}$ ) due to the photon-edges. At high temperature regime, $E_{c}(3 \omega)$ is smaller for small $m^{*}$. The parabolic term $H_{0}$ is therefore an important component in achieving strong optical nonlinearity at elevated temperature.

itly in the $n$-photon Floquet state $\psi_{n}$, as evident from Eq. (7) and (8). The effective mass therefore play a role in the NOR of R2DG albeit the fact that the subband spin-splitting is a chiefly a consequence of the Rashba term. In Fig. 5(a) and 5(b), the $m^{*}$ dependence of $E_{c}$ is plotted for $m^{*} / m$ ranging from 0.03 to 0.35 for $T=4 \mathrm{~K}$ and $T=15 \mathrm{~K}$ respectively. Since $h f_{\max }=4 m^{*} v_{F}^{2}$, the photon-edges (previously observed in Fig. 4) also occurs in the $m^{*}$ dependence plot when $m^{*}$ is just right for the onset of n-photon process (i.e. when $f_{\max }$ is a multiple of the incoming photon's frequency). This manifests as the oscillating $E_{c}$ in the $m^{*}$-dependent curve. However, the edges are more sensitively influenced by thermal excitation in the $m^{*}$ dependence curves. At $T=15 \mathrm{~K}$ [Fig. $5(\mathrm{~b})$ ], the edges are almost completely removed. In Fig. $5(\mathrm{c})$, we plot the temperature dependence of $E_{c}(3 \omega)$ at various $m^{*}$ values. In general, $E_{c}(3 \omega)$ decreases reapidly at elevated temperature [see also Fig. 3]. At low temperature, due to the $E_{c}$ oscillation of the $m^{*}$ dependence curve [Fig. 5(a)], the NOR is sensitively influenced by the $m^{*}$. However, as the oscillation is quickly washed out at elevated temperature (i.e. when $T>50 \mathrm{~K}$ ), $E_{c}$ is generally smaller at decreasing $m^{*}$. This shows that stronger optical nonlinearity is generated when $m^{*}$ is small in the intermediate to room temperature regime.

\section{DISCUSSION}

To summarize: NOR of R2DG is enhanced by two factors: (i) $v_{R}$ is large; and (ii) $m^{*}$ is small. We now discuss 
the physical significance of (i) and (ii). Factor (i) can be understood from the fact that strong NOR is a unique signature of purely linear system such as graphene $H_{K}$ with large Fermi velocity $v_{F}$. Since R2DG contains the linear component $H_{R}$, it is therefore expected to obtian larger NOR when $v_{R}$ is large. The factor (ii) is a more surprising result. Intuitively, one might expect a stronger optical nonlinerity in R2DG as the 'free' electron term vanishes $H_{0} \rightarrow 0$ such that $H_{R}$ is strongly dominant and the system becomes purely linear. Since $H_{0} \propto 1 / 2 m^{*}$, this would imply stronger optical nonlinearity at large $m^{*}$. This is, however, only true when $T \rightarrow 0$. At low temperature, the existence of sharp photon-edges does enhance the optical nonlinearity significantly. At elevated temperature, the photon-edges are completely removed and the optical nonlinearity is no longer large. On the other hand, with smaller $m^{*}$, the optical nonlinearity diminishes much slower with temperature. This corresponds to larger $H_{0}$. Therefore, it can be concluded that while large $H_{R}$ directly results in strong NOR, $H_{0}$ has rendered R2DG temperature-robust optical nonlinearity. Therefore, $H_{0}$ and $H_{R}$ are equally important in achieving strong optical nonlinearity at elevated temperature.

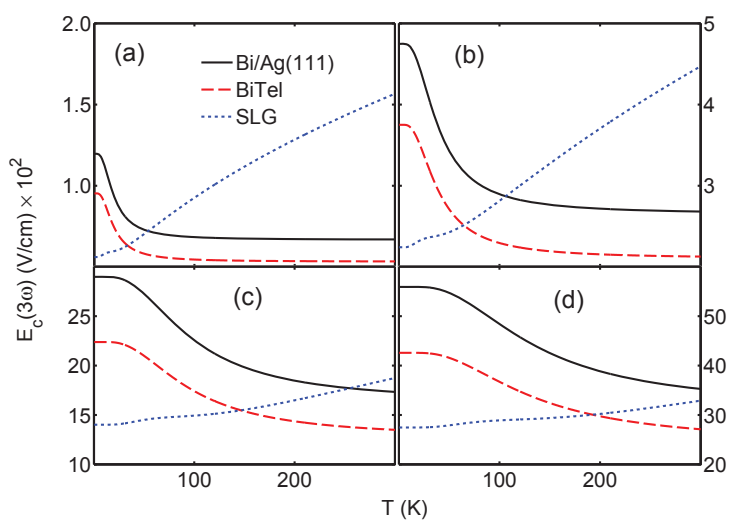

FIG. 6. (Color online) Comparison between $E_{c}(3 \omega)$ of graphene ${ }^{8}$ and R2DG in BiTeI layered semiconductor and $\mathrm{Bi} / \mathrm{Ag}(111)$ surface alloys: (a) $1 \mathrm{THz}$; (b) $2 \mathrm{THz}$; (c) $5 \mathrm{THz}$; and (d) $7 \mathrm{THz}$. Although at low temperature regime single layer graphene (SLG) has stronger optical nonlinearity (lower $E_{c}$ ), this optical nonlinearity does not survive at higher temperature. In contrast, R2DG shows stronger optical nonlinearity at elevated temperature.

Finally, we compare the NOR of R2DG with that of a single layer graphene (which is described by a linear Hamiltonian in the same form as $H_{R}$ ). Experimentally, strong RSOI in the order of $v_{R}=4.6 \times 10^{5} \mathrm{~m} / \mathrm{s}$ and $v_{R}=$ $5.8 \times 10^{5} \mathrm{~m} / \mathrm{s}$ are achieved in $\mathrm{Bi} / \mathrm{Ag}(111)$ surface state and layered polar semiconductor BiTeI respectively ${ }^{22,23}$.
Using these realistic experimental values, we calculate the important triple-frequency term $E_{c}(3 \omega)$ of these R2DG systems and comparison with graphene is made. The temperature dependence of $E_{c}(3 \omega)$ at $f=1 \mathrm{THz}$ [Fig. 6(a)], $f=2 \mathrm{THz}$ [Fig. 6(b)], $f=5 \mathrm{THz}$ [Fig. 6(c)] and $f=7 \mathrm{THz}$ [Fig. 6(d)] are plotted. For R2DG, the $E_{c}(3 \omega)$ is very sensitive to temperature variation only at low temperature regime. It rapidly decreases at intermediate temperature regime and stabilizes as $T \rightarrow 300 \mathrm{~K}$. In contrast, $E_{c}(3 \omega)$ of graphene always grows with increasing temperature ${ }^{8}$. At room temperature, and assuming that the RSOI survives at elevated temperature, the optical nonlinearity of R2DG can be 3 times stronger than graphene at $1 \mathrm{THz}$ [Fig. 6(a)]. This temperature enhanced optical nonlinearity pinpoints a major difference between the optical nonlinearity of a electronic system with purely linear Hamiltonian and that of a system with mixed linear and parabolic terms in its Hamiltonian.

\section{CONCLUSION}

In conclusion, we have calculated the inter-subband nonlinear optical spectrum of R2DG. The NOR of R2DG is found to exhibit strong NOR in the important $\mathrm{THz}$ regime. At low temperature, abrupt edges are observed in the frequency spectrum of the nonlinear optical conductivities. Such edges is a result of the cut-off effect produced by each multiple-photon terms. In contrast to an electronic system with purely linear Hamiltonian (such as graphene single layer), the critical electric field in R2DG decreases with increasing temperature. This indicates that the presence of an parabolic term $H_{0}$ induces strong optical nonlinearity at higher temperature regime. If strong RSOI in the order of $\lambda_{R}=10^{-10} \mathrm{eVm}$ can be preserved in room temperature, two-dimensional Rashba system can potentially be used as a nonlinear terahertz devices such as terahertz frequency up-converter. Recently, the R2DG in $\operatorname{Ir}(111)$ surface covered by graphene single layer has found to be well-protected from ambient atmosphere, and yet maintaining a strong RSOI in the order of $\lambda_{R} \approx 10^{-10} \mathrm{eVm}^{31}$. This provides a potential platform to experimentally probe the NOR of R2DG without the need of an ultra-vacuum environment. However, extra care has to be taken in separating out the NOR from graphene since graphene is also a strongly nonlinear medium.

\section{ACKNOWLEDGMENTS}

The work is support in part by the the Australian Research Council through a Discovery Project Grant (DP130102956). 
nova, and D. M. Treger, Science 294, 1488 (2001).

2 S. Datta and B. Das, Appl. Phys. Lett. 56, 665 (1990).

${ }^{3}$ X. F. Wang, P. Vasilopoulos, and F. M. Peeters, Phys. Rev. B65, 165217 (2002)

4 T. Koga, J. Nitta, H. Takayanagi, and S. Datta, Phys. Rev. Lett.88, 126601 (2002).

${ }^{5}$ M. Khodas, A. Shekhter and A. M. Finkel'stein Phys. Rev. Lett.92, 086602 (2004).

${ }^{6}$ G. Dresselhaus, Phys. Rev. 100, 580 (1955).

7 Y. A. Bychkov and E. I. Rashba, JETP Lett. 39, 78 (1984).

8 A. R. Wright, X. G. Xu, J. C. Cao and C. Zhang, Appl. Phys. Lett. 95, 072101 (2009).

9 S. A. Mikhailov and K. Ziegler, J. Phys.: Condens. Matter 20, 384204 (2008).

10 E. Hendry, P. J. Hale, J. Moger and A. K. Savchenko, Phys. Rev. Lett. 105? 097401 (2010).

11 G.-K. Lim et al, Nature Photon. 5, 554-560 (2011).

12 S. Shareef, Y. S. Ang, C. Zhang, J. Opt. Soc. Am. B 29, 3, 274-279 (2012)

13 Y. S. Ang, S. Sultan and C. Zhang, Appl. Phys. Lett. 97, 243110 (2010)

14 K. S. Novoselov et al, Nature 438, 197-200 (2005).

15 A. K. Geim and K. S. Novoselov, Nature Mater. 6, 183-191 (2007)
16 M. I. Katsnelson, K. S. Novoselov and A. K. Geim, Nature Phys. 2, 620 - 625 (2006).

17 F. Gao, G. Wang and C. Zhang, Nanotech. 19, 465401 (2008).

18 D. Yuan, W. Xu, Z. Zeng and F. Lu, Phys. Rev. B 72, $033320(2005)$.

19 J. A. Maytorena, C. Lpez-Bastidas and F. Mireles, Phys. Rev. B74, 235313 (2006).

20 J. Nitta, T. Akazaki and H. Takayanagi, Phys. Rev. Lett. 78, 7, 1335 - 1338 (1997)

21 D. Grundler, Phys. Rev. Lett.84, 26, 6074 - 6077 (2000).

22 C. R. Ast et al Phys. Rev. Lett.98, 186807 (2007).

${ }^{23}$ K. Ishizaka et al, Nature Mater. 10, 521-526 (2011).

${ }^{24}$ H. Sambe, Phys. Rev. A7, 6, 2203 - 2213.

25 Y. Zhou and M. W. Wu Phys. Rev. B83, 245436 (2011).

${ }^{26}$ K. Drese and M. Holthaus, Eur. Phys. J. D 5, 119 - 134 (1999).

27 S. LaShell, B. A. McDougall and E. Jensen, Phys. Rev. Lett.77, 16, 3419 - 3422 (1996).

28 K. Yaji et al, Nature Comm. 1, 17 (2010).

29 M. Koshino and T. Ando, Phys. Rev. B73, 245403 (2006).

30 E. McCann, D. S. L. Abergel and V. I. Fal'ko, Solid State Commun. 143, 110 - 115 (2007).

31 A. Varykhalov et al, Phys. Rev. Lett.108, 066804 (2012). 